\title{
Joint Beacon Power and Beacon Rate Control Based on Game Theoretic Approach in Vehicular Ad Hoc Networks
}

\author{
Hamid Garmani, Driss Ait Omar, Mohamed El Amrani, Mohamed Baslam, and Mostafa Jourhmane
}

\begin{abstract}
In vehicular ad hoc networks (VANETs), each vehicle broadcasts its information periodically in its beacons to create awareness for surrounding vehicles aware of their presence. But, the wireless channel is congested by the increase beacons number, packet collision lost a lot of beacons. This paper tackles the problem of joint beaconing power and a beaconing rate in VANETs. A joint utilitybased beacon power and beacon rate game are formulated as a non-cooperative game and a cooperative game. A three distributed and iterative algorithm (Nash Seeking Algorithm, Best Response Algorithm, Cooperative Bargaining Algorithm) for computing the desired equilibrium is introduced, where the optimal values of each vehicle beaconing power and beaconing rate are simultaneously updated at the same step. Extensive simulations show the convergence of a proposed algorithm to the equilibrium and give some insights on how the game parameters may vary the game outcome. It is demonstrated that the Cooperative Bargaining Algorithm is a fast algorithm that converges the equilibrium.
\end{abstract}

Index Terms-. Beacon rate, Beacon power, Non-cooperative game, Cooperative game, VANETs, Game theory, Nash equilibrium, Nash bargaining solution.

\section{INTRODUCTION}

$\mathrm{V}$ VANETs is a new paradigm of wireless communications that aim to exploit the recent advances in wireless devices technology to enable intelligent inter-vehicle communication. The appearance of VANETs has been becoming an interesting field for the traffic research community during the last decades. VANETs provides a new trend for Intelligent Transportation Systems such as public transport management [1], and improve security in transportation to reduce the number of disasters. Various types of safety have been designed for VANETs, including emergency alert, accident notification, curve alert, file-sharing, internet, and advertisements.

This paragraph of the first footnote will contain the date on which you submitted your paper for review.

Authors are with the Information Processing and Decision Support Laboratory, Faculty of Sciences and Technics, University Sultan Moulay Slimane, Beni Mellal, Morocco (e-mails: garmani.hamid@gmail.com, aitomard@gmail.com, med.el.amran@gmail.com, baslam.med@gmail.com, jourhman@hotmail.com).
Enhance security is achieved by Basic Safety Messages (BSMs) exchanged between vehicles in VANETs, the BSMs are called beacons. Vehicles periodically broadcast beacons within the network to inform other vehicles of their situation (vehicle nodes position, speed, and direction information). On the other hand, beacons or safety messages are broadcasted in case of emergencies, such as collisions, accidents, and road surface collapse. In dense vehicular networks, a high number of beacons get lost, and congestion in the channel load result because of the growth in beaconing rates, and thus, degrades vehicles' awareness and the accuracy of the safety of vehicles. Channel congestion is a critical factor that leads to delayed or failed messages delivery. With higher vehicle density, it is not clear if the channel capacity will be sufficient to support the data load generated by beacons. Therefore, the development of effective congestion control strategies for VANETs is of utmost importance and has been an area of intense research interest in recent years.

The modelization of analytical models to study the behavior of the vehicle in VANETs is a challenge that gets an increasing interest of researchers. Several models have been proposed to analyze the VANET performance to suggest suitable solutions to VANETs. Congestion control is a challenge in computer networks. The metric used to evaluate congestion control are fairness between the vehicle, the time needed for the convergence, and oscillation size [2]. Congestion control in VANETs should operate in a distributed manner without involving any infrastructure. Due to the highly dynamic nature of VANETs, the convergence time of the control mechanism must be minimal.

Several work used game theory in wireless networks [3] [4] [5] [6] [7] [8]. The authors in [9] proposed a beacon power control algorithm; every player calculates the maximum beaconing power to achieve the maximum communication power and keeps the Channel Busy Ratio (CBR) under a threshold. In [10], the authors study the performance of a multihop broadcast protocol in VNETs safety by designing a generic probabilistic forwarding scheme and proposing an analytical model to study the performance of the proposed model. The authors in [11] provide a mechanism to find the optimal beacon rates founded on the maximization of the utility function and show the impact of the beacon rate on the performance of the network. In [12], the author studied a dynamic congestion control mechanism as a means of broadcasting BSM, and to guarantee the reliable and timely delivery of messages to all 
neighbors in a network. The authors in [13] used the tabu search algorithm with multi-channel allocation capability to reduce the time delay and jitter for improving the quality of service in VANET. In [10], the authors proposed a vehicle mobility prediction founded beacon rate adaptation approach, where each vehicle uses the prediction module to get the situation of their neighbors in real-time. The authors in [14] studied the competition among vehicles in beaconing power as a noncooperative game. In [15] the authors used the non-cooperative game for designing a beacon rate control mechanism. The authors proved the uniqueness of the Nash equilibrium point and proposed a distributed method is used to find the equilibrium point. In this paper, we utilize a non-cooperative game and the cooperative game to study the joint control beaconing rate and beaconing power in VANETs. We propose three algorithms for learning joint beaconing rate and beaconing power at Nash equilibrium and Nash bargaining solution.

In this paper, a fair and stable joint beaconing power and beaconing rate problem in VANETs are formulated and solved based on the non-cooperative games and cooperative game. The incentive and objective of the proposed approach are finding the vehicle beaconing power and beaconing rate in a distributed manner to decrease the number of losses of beacons. The theory of supermodular games and the Nash bargaining solution are used to solve the corresponding optimization problem. We prove the existence of the Nash equilibrium point in the noncooperative game. Furthermore, we implement three learning algorithms that find the equilibrium point in a distributed manner by adjusting beaconing rates and beaconing powers jointly in a single step. Performance evaluation shows the convergence of the proposed algorithm to the equilibrium beaconing power and the beaconing equilibrium rate, and show the impact of system parameters on vehicle strategies. Also, it is revealed that the proposed cooperative game algorithm is the best choice for the vehicle to control the beaconing rate and beaconing power.

The rest of this paper is organized as follows. In Section II, we describe the proposed model. In Section III, we present the non-cooperative game formulation and the price of anarchy. In Section IV, we present a cooperative game. Then, we present the Performance evaluation in Section V. Finally, in Section VI conclusions.

\section{SYSTEM MODEL}

The utility function of each vehicle is the difference between revenue and fees. Accordingly, the payoff of the vehicle $i$ can be written as:

$$
\begin{gathered}
U_{i}=a_{i} \log \left(r_{i}+p_{i}+1\right)-c_{i} p_{i} C B R_{i}\left(p_{i}, r_{i}, p_{-i}, r_{-i}\right)-\left(C_{s_{i}}+\right. \\
\left.C_{p_{i}} p_{i}+C_{r_{i}} r_{i}\right)
\end{gathered}
$$

where $a_{i}$ and $c_{i}$ are two positive parameters. $C B R_{i}\left(p_{i}, r_{i}, p_{-i}, r_{-i}\right)$ is the channel busy ratio that vehicle $i$ senses, and it is a function of all vehicle beaconing rates and beaconing power, where $p_{-i}=\left(p_{1}, \ldots, p_{i-1}, p_{i+1}, \ldots, p_{\mathrm{N}}\right)$. The term $a \log \left(r_{i}+p_{i}+1\right)$ is the revenue of vehicle $i$; it is an increasing function with respect to beaconing rate and beaconing power. A logarithmic function has been used because it is increasing and has excellent concavity properties. Thus, the vehicle with lower beaconing power and their beaconing rate has more incentive to increase their beaconing power and their beaconing rate. The second term $c_{i} p_{i} C B R_{i}\left(p_{i}, r_{i}, p_{-i}, r_{-i}\right)$, is the congestion cost. It indicates that a vehicle should pay higher costs at higher congestions, which discourages the vehicles from using a high beacon rate and high beacon power. The third term $C_{s_{i}}+C_{p_{i}} p_{i}+C_{r_{i}} r_{i}$ is the energy consumed to send beacons and to switch the state of the transceiver. $C_{s_{i}}$ is the energy consumed for switching the state of the transceiver, $C_{p_{i}}$ is the energy consumed for sending beacons with power $p_{i}$, and $C_{r_{i}}$ is the energy consumed for sending beacons with a rate $r_{i}$.

Then, we define $C B R_{i}\left(p_{i}, r_{i}, p_{-i}, r_{-i}\right)$ as that in [16] by

where

$$
C B R_{i}\left(p_{i}, r_{i}, p_{-i}, r_{-i}\right)=\sum_{j=1}^{N} h_{i j} r_{j}
$$

$$
\begin{aligned}
& h_{i j}=T_{\text {frame }} \times \frac{\Gamma\left(m, m \frac{C_{T t}}{\Omega_{i j}}\right)}{\Gamma(m)} \\
& \Omega_{i j}=\frac{p_{j} \lambda^{2}}{(4 \pi)^{2} d_{i j}^{\gamma}}
\end{aligned}
$$

$\Gamma$ is the gamma function, $\Gamma(.,$.$) is the upper incomplete gamma$ function, $C_{T t}$ is the threshold power level of carrier sense, $p_{j}$ is the $B S M$ transmit power of vehicle $j, d_{i j}$ is the distance between $j$ th and $i$ th vehicles, $m$ is Nakagami fading parameter, $\lambda$ is the wavelength, $\gamma$ is the path loss exponent, $r_{j}$ is the beaconing rate of vehicles $j$, and $T_{\text {frame }}$ is the time needed to transmit a beacon message.

Equation (2) indicates that the channel load experienced by vehicle $i$ is the weighted sum of the beaconing rate of all the other vehicles $\sum_{j=1}^{N} h_{i j} r_{j}$. The channel load also depends on various parameters such as channel fading, the time needed to transmit a beacon message, and the distance of other vehicles. The coefficients $h_{i j}$ defined in (3), represents the action of these parameters in the channel load sensed by vehicle $i$.

\section{A NON-COOPERATIVE GAME FORMULATION}

Let $G=\left[\mathcal{N},\left\{R_{i}, P_{i}\right\},\left\{U_{i}().\right\}\right]$ denote the non-cooperative beaconing rate and beaconing power game (NRPG), where $\mathcal{N}=\{1, \ldots, N\}$ is the index set identifying the vehicle, $P_{i}$ is the beaconing power strategy set of vehicle $i, R_{i}$ is the beaconing rate strategy set of vehicle $i$, and $U_{i}($.$) is the utility function of$ vehicle $i$ defined in Equation (1). We assume that the strategy spaces $R_{i}$ and $P_{i}$ of each vehicle $i$ are compact and convex sets with maximum and minimum constraints, for any given vehicle $i$ we consider as strategy spaces the closed intervals $R_{i}=\left[\underline{r}_{i}, \bar{r}_{i}\right]$ and $P_{i}=\left[\underline{p}_{i}, \bar{p}_{i}\right]$. Let the beaconing power vector $\mathbf{p}=$ $\left(p_{1}, \ldots, p_{N}\right)^{T} \in P^{N}=P_{1} \times P_{2} \times \ldots \times P_{N}$, beaconing rate vector $\mathbf{r}=\left(r_{1}, \ldots, r_{N}\right)^{T} \in R^{N}=R_{1} \times R_{2} \times \ldots \times R_{N}$. 
Definition 1 The strategy vector $\left(\boldsymbol{p}^{*}, \boldsymbol{r}^{*}\right)=$ $\left(p_{1}^{*}, p_{2}^{*}, \ldots, p_{N}^{*}, r_{1}^{*}, r_{2}^{*}, \ldots, r_{N}^{*}\right)$ is a Nash equilibrium of the $N R P G G=\left[\mathcal{N},\left\{R_{i}, P_{i}\right\},\left\{U_{i}(.,).\right\}\right]$ if

$$
\begin{aligned}
& \forall\left(i, r_{i}, p_{i}\right) \in\left(\mathcal{N}, R_{i}, P_{i}\right), \\
& U_{i}\left(p_{i}^{*}, r_{i}^{*}, \mathbf{p}_{-i}^{*}, \mathbf{r}_{-i}^{*}\right) \geq U_{i}\left(p_{i}, r_{i}, \mathbf{p}_{-i}^{*}, \mathbf{r}_{-i}^{*}\right)
\end{aligned}
$$

Definition 2 The game $G$ is submodular if she satisfies the following conditions:

- $S_{i}=P_{i} \times R_{i}$ is a compact subset of Euclidean space.

- $U_{i}\left(p_{i}, r_{i}\right), p_{i} \in P_{i}, r_{i} \in R_{i}$ is smooth and: $\checkmark$ submodular in $\left(p_{i}, r_{i}\right)$ for fixed $\left(\mathbf{p}_{-i}, \mathbf{r}_{-i}\right)$ i.e.,

$$
\frac{\partial^{2} U_{i}}{\partial p_{i} \partial r_{i}} \leq 0
$$

$$
\begin{array}{r}
\checkmark \text { Has non-increasing } \\
\left\{\left(p_{i}, r_{i}\right),\left(\mathbf{p}_{-i}, \mathbf{r}_{-i}\right)\right\}, \text { i.e. }, \\
\frac{\partial^{2} U_{i}}{\partial r_{i} \partial r_{j}} \leq 0, \quad \forall j \neq i
\end{array}
$$

differences

given that

$$
\frac{\partial^{2} U_{i}}{\partial r_{i} \partial p_{j}}=0, \forall j \neq i
$$

Theorem 1 The utility function $U_{i}(\boldsymbol{p}, \boldsymbol{r})$ is submodular in $\left(p_{i}, r_{i}\right)$ for fixed $\left(\boldsymbol{p}_{-i}, \boldsymbol{r}_{-i}\right)$.

Proof: The second-order partial derivative utility function is written as:

$$
\frac{\partial^{2} U_{i}}{\partial p_{i} \partial r_{i}}=-\frac{a_{i}}{\left(1+r_{i}+p_{i}\right)^{2}}-c_{i} h_{i i} \leq 0
$$

then the utility function $U_{i}(\mathbf{p}, \mathbf{r})$ is submodular in $\left(p_{i}, r_{i}\right)$ for each fixed $\left(\mathbf{p}_{-i}, \mathbf{r}_{-i}\right)$.

Theorem 2 The utility function $U_{i}(\boldsymbol{p}, \boldsymbol{r})$ has non-increasing differences in $\left\{\left(p_{i}, r_{i}\right),\left(\boldsymbol{p}_{-i}, \boldsymbol{r}_{-i}\right)\right\}$.

Proof: The second partial derivative of the utility function is

$$
\frac{\partial^{2} U_{i}}{\partial r_{i} \partial r_{j}}=0
$$

and

$$
\frac{\partial^{2} U_{i}}{\partial r_{i} \partial p_{j}}=0
$$

Then the utility function $U_{i}(\mathbf{p}, \mathbf{r})$ has non-increasing differences in $\left\{\left(p_{i}, r_{i}\right),\left(\mathbf{p}_{-i}, \mathbf{r}_{-i}\right)\right\}$.

Based on theorems 1, theorems 2, and definition 2, we conclude the following theorems.

Theorem 3 The NRPG $G$ is submodular in $\left(p_{i}, r_{i}\right)$ for all $i \in$ $\mathcal{N}$.

Based on theorem 3, the game $G$ is a submodular game, and the set of its Nash equilibrium points is nonempty. Therefore, the following holds:

Theorem 4 The NRPG game $G=\left[\mathcal{N},\left\{R_{i}, P_{i}\right\},\left\{U_{i}(\mathbf{p}, \mathbf{r})\right\}\right]$ has at least one Nash equilibrium [6], which is defined as:

$$
\left(p_{i}^{*}, r_{i}^{*}\right)=\arg \max _{p_{i} \in P_{i}, r_{i} \in R_{i}} U_{i}(\mathbf{p}, \mathbf{r})
$$

The following theorem proves the uniqueness of the Nash equilibrium point.
Theorem 5 The unique Nash equilibrium point of the NRPG G is given by:

s.t.

$$
\left(p_{i}^{*}, r_{i}^{*}\right)=\arg \max _{p_{i} \in P_{i}, r_{i} \in R_{i}} U_{i}(\mathbf{p}, \mathbf{r})
$$

$$
\left.\frac{\partial U_{i}(\mathbf{p}, \mathbf{r})}{\partial p_{i}}\right|_{p_{i}=p_{i}^{*}}=0 \quad \text { and }\left.\quad \frac{\partial U_{i}(\mathbf{p}, \mathbf{r})}{\partial r_{i}}\right|_{r_{i}=r_{i}^{*}}=0
$$

and

$$
\left(p_{i}, r_{i}\right) J\left(p_{i}, r_{i}\right)\left(p_{i}, r_{i}\right)^{T} \leq 0, \quad \forall p_{i} \in P_{i}, \quad \forall r_{i} \in R_{i}
$$

where $J=\left(\begin{array}{ll}\frac{\partial^{2} U_{i}}{\partial p_{i}^{2}} & \frac{\partial^{2} U_{i}}{\partial p_{i} \partial r_{i}} \\ \frac{\partial^{2} U_{i}}{\partial p_{i} \partial r_{i}} & \frac{\partial^{2} U_{i}}{\partial r_{i}^{2}}\end{array}\right)$ is the Hessian matrix at point $\left(p_{i}, r_{i}\right)$.

Proof: The conditions of the first-order partial derivatives (13) determine the stationary points of the utility function $U_{i}(\mathbf{p}, \mathbf{r})$, which can either be a maximum, a minimum or a saddle point. The condition (14) is necessary to find the global maximum of the utility function.

$$
\begin{gathered}
J=\left(\begin{array}{cc}
\frac{\partial^{2} U_{i}}{\partial p_{i}^{2}} & \frac{\partial^{2} U_{i}}{\partial p_{i} \partial r_{i}} \\
\frac{\partial^{2} U_{i}}{\partial p_{i} \partial r_{i}} & \frac{\partial^{2} U_{i}}{\partial r_{i}^{2}}
\end{array}\right) \\
=\left(\begin{array}{cc}
-\frac{a_{i}}{\left(1+r_{i}+p_{i}\right)^{2}} & -\frac{a_{i}}{\left(1+r_{i}+p_{i}\right)^{2}}-c_{i} h_{i i} \\
-\frac{a_{i}}{\left(1+r_{i}+p_{i}\right)^{2}}-c_{i} h_{i i} & -\frac{a_{i}}{\left(1+r_{i}+p_{i}\right)^{2}}
\end{array}\right)
\end{gathered}
$$

Thus,

$$
\begin{gathered}
\left(p_{i}, r_{i}\right) J\left(p_{i}, r_{i}\right)\left(p_{i}, r_{i}\right)^{T}=-\frac{a_{i} p_{i}^{2}}{\left(1+r_{i}+p_{i}\right)^{2}}-\frac{a_{i} r_{i}^{2}}{\left(1+r_{i}+p_{i}\right)^{2}} \\
-\frac{a_{i} p_{i}^{2}}{\left(1+r_{i}+p_{i}\right)^{2}}-c_{i} h_{i i} p_{i}^{2}-\frac{a_{i} r_{i}^{2}}{\left(1+r_{i}+p_{i}\right)^{2}}-c_{i} h_{i i} r_{i}^{2} \leq 0
\end{gathered}
$$

Then, the Hessian matrix $J$ is negative definite.

Since it is hard to get the analytical result of the system (13), we use an iterative and distributed algorithm that finds the unique Nash equilibrium point $\left(\mathbf{p}^{*}, \mathbf{r}^{*}\right)$. This algorithm is defined as follows.

\section{A. Iterative Nash Equilibrium Algorithm}

In this section, based on our previous analysis, we introduce two distributed and iterative learning processes that convergence toward the Nash equilibrium point of NRPG. The best response algorithm is known to reach equilibria for Smodular games, by exploiting the monotonicity of the best response functions. Each player fixes its desirable strategies to maximize its profit. Then, each player can observe the policy taken by its competitors in previous rounds and input them in its decision process to update its policy. Then, it becomes natural to accept the Nash equilibrium as the attractive point of the game. Yet, the best response algorithm requires perfect rationality and complete information, which is not practical for real-world applications and may increase the signaling load as well. Therefore, we propose an adaptive distributed learning framework to discover equilibria for the activation game based on the "Nash Seeking Algorithm" with stochastic state- 
dependent payoffs for continuous actions. Algorithm 1 summarizes the best response learning steps that each player has to perform to discover its Nash equilibrium strategy.

Nash seeking algorithm is one of the most Known learning schemes. It is a discrete-time learning algorithm, using sinus perturbation, for continuous action games where each vehicle has only a numerical realization of the payoff at each time. At each iteration $t$, the vehicle $i$ chooses its beaconing power and beaconing rate and obtains from the environment the realization of its payoff. The improvement of the strategy is based on the current observation of the realized payoff and previously chosen strategies. Hence, we say vehicles learn to play an equilibrium, if after a given number of iterations, the strategy profile converges to an equilibrium strategy. The proposed learning framework has the following parameters: $\phi_{i}$ and $\phi_{i}^{\prime}$ are the perturbation phase, $z_{i}$ and $z_{i}^{\prime}$ are the growth rate, $b_{i}$ and $b_{i}^{\prime}$ are the perturbation amplitude, and $\Omega_{i}$ and $\Omega^{\prime}{ }_{i}$ are the perturbation frequency. This procedure is repeated for the window T. Algorithm 2 summarizes the Nash seeking algorithm learning steps that vehicle $i$ has to perform in order to discover its Nash equilibrium beaconing power and beaconing rate.
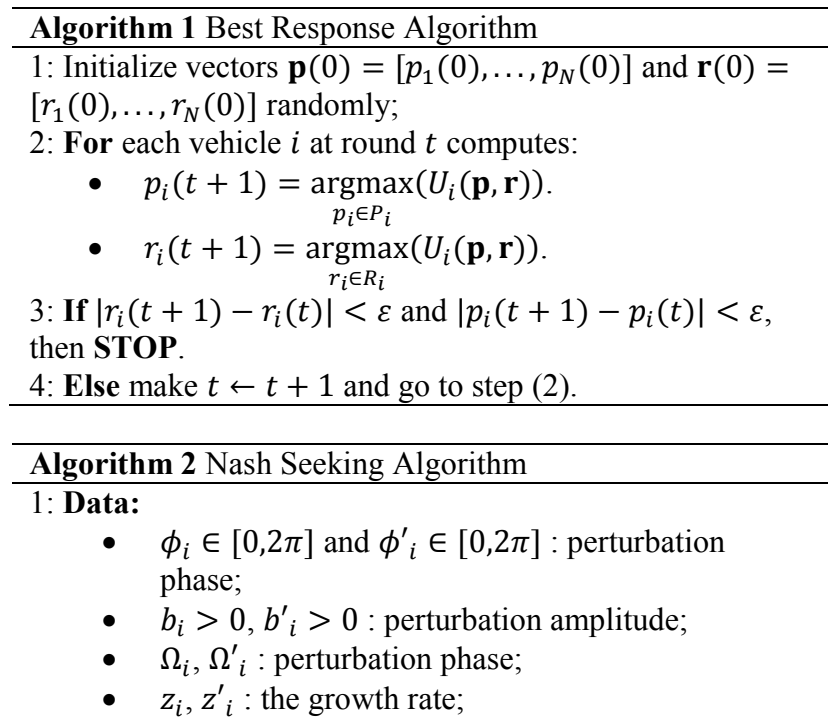

2: Result: Equilibrium beaconing power $p_{i}$ and Equilibrium beaconing rate $r_{i}$

3: Initialization:

4: Assign a value for $\tau_{i, 0}^{*}, \varsigma_{i, 0}^{*}, p_{i, 0}^{*}$ and $r_{i, 0}^{*}$ for $i=1,2, \ldots, N$;

5: Learning pattern: For each iteration $t$ :

6: Observes the payoff $U_{i, t}$ and estimates $\tau_{i, t+1}^{*}$ and $\zeta_{i, t+1}^{*}$ using

- $\tau_{i, t+1}^{*}=\tau_{i, t}^{*}+t^{*} z_{i} b_{i} \sin \left(\Omega_{i} t^{*}+\phi_{i}\right) U_{i, t}$

- $S_{i, t+1}^{*}=s_{i, t}^{*}+t^{*} z^{\prime}{ }_{i} b_{i}^{\prime} \sin \left(\Omega^{\prime}{ }_{i} t^{*}+\phi_{i}^{\prime}\right) U_{i, t}$;

7: Update beaconing rate $r_{i}$ and beaconing power $p_{i}$ using the following rules

- $p_{i, t+1}^{*}=\tau_{i, t+1}^{*}+b_{i} \sin \left(\Omega_{i} t^{*}+\phi_{i}\right)$;

- $r_{i, t+1}^{*}=\varsigma_{i, t+1}^{*}+b_{i}^{\prime} \sin \left(\Omega_{i}^{\prime} t^{*}+\phi_{i}^{\prime}\right)$;

\section{B. Price of Anarchy}

The price of anarchy (PoA) is defined as the ratio between the performance measures of the worst equilibrium and the optimal outcome. A PoA close to 1 indicates that the equilibrium is approximately socially optimal, and thus the consequences of selfish behavior are relatively benign.

In [17], we measure the loss of efficiency due to actors' selfishness as the quotient between the social welfare obtained at the Nash equilibrium and the maximum value of the social welfare:

$$
P o A=\frac{\min _{p, r} W_{N E}(\mathbf{p}, \mathbf{r})}{\max _{p, r} W(\mathbf{p}, \mathbf{r})}
$$

where $W(p, r)=\sum_{i=1}^{N} U_{i}(\mathbf{p}, \mathbf{r})$ is the social welfare function and $W_{N E}\left(\mathbf{p}^{*}, \mathbf{r}^{*}\right)=\sum_{i=1}^{N} U_{i}(\mathbf{p}, \mathbf{r})$ is a sum of utilities of all players at Nash Equilibrium.

\section{COOPERATIVE GAME}

The Nash bargaining game [18] is a cooperative game in which players have a mutual agreement for cooperation in order to obtain a higher payoff compared to the non-cooperative case. Let $\mathcal{U}$ be a closed and convex subset of $\mathbb{R}^{N}$ that represents the set of feasible payoff allocations that the players can get if they all cooperate. Suppose $\left\{U_{i} \in \mathcal{U} \mid U_{i} \geq U_{i}^{\min }, \forall i \in \mathcal{N}\right\}$ is a nonempty bounded set. Define $\mathbf{U}^{\text {min }}=$ $\left(U_{1}^{\text {min }}, U_{2}^{\text {min }}, \ldots, U_{N}^{\text {min }}\right)$, then the pair of $\left(\mathcal{U}, \mathbf{U}^{\text {min }}\right)$ constructs a $K$-player bargaining game. Here, we define the Pareto efficient point [19], where a player can not find another point that improves the utility of all the players at the same time.

Definition 3 A strategy profile $\left(\boldsymbol{p}^{*}, \boldsymbol{r}^{*}\right)=$ $\left(p_{1}^{*}, p_{2}^{*}, \ldots, p_{N}^{*}, r_{1}^{*}, r_{2}^{*}, \ldots, r_{N}^{*}\right)$ is Pareto-optimal if and only if there is no other strategy profile $(\mathbf{p}, \mathbf{r})$ such that $U_{i}(\mathbf{p}, \mathbf{r}) \geq$ $U_{i}\left(\mathbf{p}^{*}, \mathbf{r}^{*}\right), \forall i \in \mathcal{N}$, and $U_{i}(\mathbf{p}, \mathbf{r})>U_{i}\left(\mathbf{p}^{*}, \mathbf{r}^{*}\right), \exists i \in \mathcal{N}$, i.e., there exists no other strategies that lead to superior performance for some players without causing inferior performance for some other players [19].

There may be an infinite number of Pareto optimal points in a game of multi-players. Thus, we must address how to select a Pareto point for a cooperative bargaining game. We need a criterion to select the best Pareto point of the system. A possible criterion is the fairness of resource allocation. Notably, the fairness of bargaining games is a Nash bargaining solution, which can provide a unique and fair Pareto optimal point under the following axioms.

Definition $4 \bar{r}$ is a Nash bargaining solution in $\mathcal{U}$ for $\mathbf{U}^{\text {min }}$ i.e., $\bar{r}=\mathcal{H}\left(\mathcal{U}, \mathbf{U}^{\text {min }}\right)$, if the following axioms are satisfied [19].

- Individual rationality: $\bar{r}_{i} \geq U_{i}^{\min }, \bar{r}_{i} \in \bar{r}, i \in \mathcal{N}$.

- Feasibility: $\bar{r} \in \mathcal{U}$.

- Pareto Optimality: $\bar{r}$ is Pareto optimal.

- Independence of Irrelevant Alternatives: If $\bar{r} \in \mathcal{U}^{\prime} \subset$ $\mathcal{U}, \bar{r}=\mathcal{H}\left(\mathcal{U}, \mathbf{U}^{\text {min }}\right)$, then $\bar{r}=\mathcal{H}\left(\mathcal{U}^{\prime}, \mathbf{U}^{\text {min }}\right)$.

- Independence of Linear Transformations: For any linear scale transformation $\Theta, \Theta\left(\mathcal{H}\left(\mathcal{U}, \mathbf{U}^{\text {min }}\right)\right)=$ $\mathcal{H}\left(\Theta(\mathcal{U}), \Theta\left(\mathbf{U}^{\text {min }}\right)\right)$.

- Symmetry: If $\mathcal{U}$ is invariant under all exchanges of players, that is $\mathcal{H}_{i}\left(\mathcal{U}, \mathbf{U}^{\text {min }}\right)=\mathcal{H}_{j}\left(\mathcal{U}, \mathbf{U}^{\text {min }}\right), \forall i, j$. 
Joint Beacon Power and Beacon Rate Control Based on Game Theoretic Approach in Vehicular Ad Hoc Networks
Theorem 6 A unique and fair Nash bargaining solution $\mathbf{x}^{*}=$ $\left(\mathbf{p}^{*}, \mathbf{r}^{*}\right)$ that satisfies all the axioms in Definition 4 can be obtained by maximizing a product term as follows:

$$
\boldsymbol{x}^{*}=\underset{p_{i} \in P_{i}, r_{i} \in R_{i}}{\operatorname{argmax}} \prod_{i=1}^{N} U_{i}(\mathbf{p}, \mathbf{r})
$$

Proof: The proof of the theorem 6 is omitted due to space limitations. A similarly detailed proof can be found in [18].

Our work aims to maximize utility functions while decreasing the number of losses beacons. Therefore, the corresponding cooperative Nash bargaining game-theoretic power and rate control problem for vehicle underlying the communication system can be formulated as:

$$
\begin{aligned}
& \text { P1: } \max _{p_{i} \in P_{i}, r_{i} \in R_{i}} \prod_{i=1}^{N} U_{j}(\mathbf{p}, \mathbf{r}) \\
& \text { s.t. } \quad\left\{\begin{array}{l}
C 1: 0 \leq p_{i} \leq p_{i}^{\text {max }} \\
\mathrm{C} 2: 0 \leq r_{i} \leq r_{i}^{\text {max }}
\end{array}\right.
\end{aligned}
$$

where constraint $C 1$ limits the beaconing power of vehicle $i$ to be below $p_{i}^{\max }$ and $C 2$ limits the beaconing rate of vehicle $i$ to be below $r_{i}^{\max }$.

Lemma 1 Define $V_{i}(\mathbf{p}, \mathbf{r}) \triangleq \ln \left(U_{i}(\mathbf{p}, \mathbf{r})\right), \quad i \in \mathcal{N}$. These objective functions are concave and injective, which satisfy all the Nash axioms in Definition 4.

Proof: The proof of theorem 5 shows that the Hessian matrix of the utility function $U_{i}(\mathrm{p}, \mathrm{r})$ is negatively define. Then, the utility function $U_{i}(\mathrm{p}, \mathrm{r})$ is strictly concave with regard to the 2tuple $\left(p_{i}, r_{i}\right)$. Subsequently, $V_{i}(\mathbf{p}, \mathbf{r})=\ln \left(U_{i}(\mathbf{p}, \mathbf{r})\right)$ is also concave in $\left(p_{i}, r_{i}\right)$. Therefore, $V_{i}(\mathbf{p}, \mathbf{r})$ defined above satisfies all the axioms required by Definition 4 and Theorem 6 .

According to Theorem 6 and Lemma 1, the unique Nash bargaining equilibrium with fairness can be found over the strategy space. Then, taking advantage of the increasing property of the logarithmic function, the optimization problem P1 can be rewritten as:

$$
\begin{array}{r}
\text { P2: } \max _{p_{i} \in P_{i}, r_{i} \in R_{i}} \sum_{i=1}^{N} V_{i}(\mathbf{p}, \mathbf{r})=\max _{p_{i} \in P_{i}, r_{i} \in R_{i}} \sum_{i=1}^{N} U_{i}(\mathbf{p}, \mathbf{r}) \\
\text { s.t. } \quad\left\{\begin{array}{l}
C 1: 0 \leq p_{i} \leq p_{i}^{\text {max }} \\
C 2: 0 \leq r_{i} \leq r_{i}^{\text {max }}
\end{array}\right.
\end{array}
$$

\section{A. Solution of the Cooperative Gam}

Herein, we derive the unique equilibrium by solving the constrained optimization problem in (20) utilizing the method of Lagrange multipliers [20]. Introducing Lagrange multipliers $\left\{\chi_{i}^{i t e}\right\}_{i=1}^{N}$ and $\left\{\psi_{i}^{i t e}\right\}_{i=1}^{N}$ for the multiple constraints, the Lagrangian of problem (20) can equivalently be solved by maximizing the following expression:

$\mathcal{F}\left(\mathbf{p}, \mathbf{r},\left\{\chi_{i}^{i t e}\right\}_{i=1}^{N},\left\{\psi_{i}^{i t e}\right\}_{i=1}^{N}\right)=\sum_{i=1}^{N}\left(a_{i} \log \left(r_{i}+p_{i}+1\right)-\right.$

$\left.c_{i} p_{i} C B R_{i}(\mathbf{p}, \mathbf{r})-\left(C_{s_{i}}+C_{p_{i}} p_{i}+C_{r_{i}} r_{i}\right)-\chi_{i} p_{i}-\psi_{i} r_{i}\right)$

Based on the standard optimization methods and the KarushKuhn-Tucker conditions, the beaconing power of vehicle $i$ can be obtained by taking the first derivative of (21) with respect to $p_{i}$, which is expressed as follows:

$$
\frac{\partial \mathcal{F}}{\partial p_{i}}=\frac{a_{i}}{1+p_{i}+r_{i}}-c_{i} C B R(\mathbf{p}, \mathbf{r})-C_{p_{i}}-\chi_{i}
$$

Letting $\frac{\partial \mathcal{F}}{\partial p_{i}}=0$ we get,

$$
p_{i}^{*}=\frac{a_{i}}{c_{i} C B R(\mathbf{p}, \mathbf{r})+C_{p_{i}}+\chi_{i}^{*}}-1-r_{i}^{*}
$$

Meanwhile, the beaconing rate of vehicle $i$ can be obtained by taking the first derivative of (21) with respect to $r_{i}$ as

$$
\frac{\partial \mathcal{F}}{\partial r_{i}}=\frac{a_{i}}{1+p_{i}+r_{i}}-c_{i} h_{i i}-C_{r_{i}}-\psi_{i}
$$

Let (24) equals to zero, then we get

$$
r_{i}^{*}=\frac{a_{i}}{c_{i} h_{i i}+C_{r_{i}}+\psi_{i}^{*}}-1-p_{i}^{*}
$$

In this work, we employ the fixed-point technique to derive an iterative procedure that updates the beaconing rate and beaconing power control decisions, which can be given as:

$$
\begin{aligned}
& p_{i}^{i t e+1}=\left[\frac{a_{i}}{c_{i} C B R(\mathbf{p}, \mathbf{r})+C_{p_{i}}+\chi_{i}^{i t e}}-1-r_{i}^{i t e}\right]_{0}^{p_{i}^{\max }} \\
& r_{i}^{i t e+1}=\left[\frac{a_{i}}{c_{i} h_{i i}+C_{r_{i}}+\psi_{i}^{i t e}}-1-p_{i}^{i t e}\right]_{0}^{r_{i}^{\max }}
\end{aligned}
$$

\section{B. Update of the Lagrange Multipliers}

The Lagrange multipliers $\left\{\chi_{i}^{i t e}\right\}_{i=1}^{N}$ and $\left\{\psi_{i}^{i t e}\right\}_{i=1}^{N}$ need to be updated to guarantee the fast convergence property. Several practical approaches can be employed in the update of Lagrange multipliers. In this paper, the sub-gradient technique is utilized to update the multipliers, as formulated as follows:

$$
\left\{\begin{array}{l}
\psi_{i}^{i t e+1}=\left[\psi_{i}^{i t e}-\alpha^{i t e} p_{i}^{i t e+1}\right]^{+} \\
\chi_{i}^{i t e+1}=\left[\chi_{i}^{i t e}-\alpha^{i t e} r_{i}^{i t e+1}\right]^{+}
\end{array}\right.
$$

where $(x)^{+}=\max (0, x), \beta$ denotes the step size of iteration ite (ite $\in\left\{1,2, \ldots, L_{\max }\right\}$ and $L_{\max }$ denotes the maximum number of iterations.

\section{Iterative Nash Bargaining Algorithm}

In this section, a distributed algorithm is proposed as an implementation of our cooperative bargaining beaconing rate and beaconing power control solution. The proposed iterative Algorithm 3 will guarantee convergence by using the subgradient method.

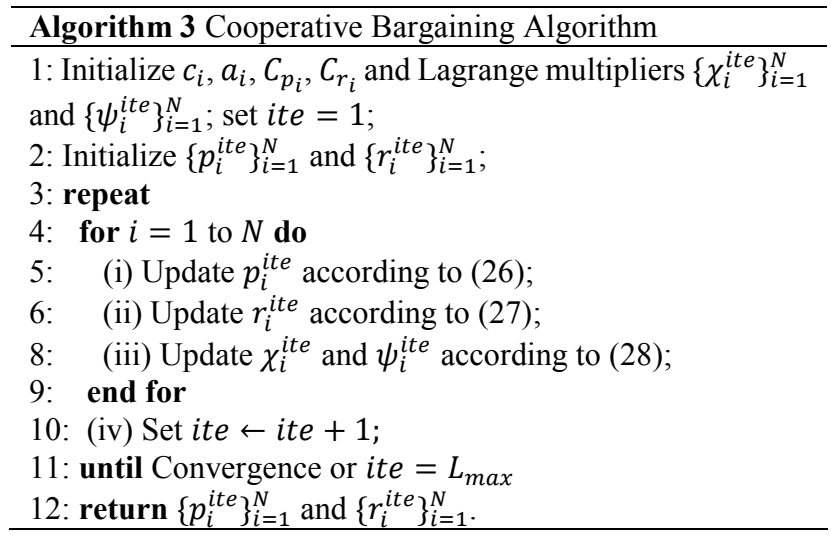




\section{PERFORMANCE EVALUATION}

Extensive experiments have been conducted toward investigating the following issues: (1) what is the number of iterations required by the proposed algorithm to converge toward the equilibrium beaconing rate and equilibrium beaconing power; (2) what is the fast algorithm that converges toward the equilibrium strategies; (3) In what way could system parameters affect the beaconing equilibrium rate and the equilibrium beaconing power? In this section, we demonstrate these experimental results by considering the previous expressions of the utility function. As an illustration, we consider a scenario with two vehicles.

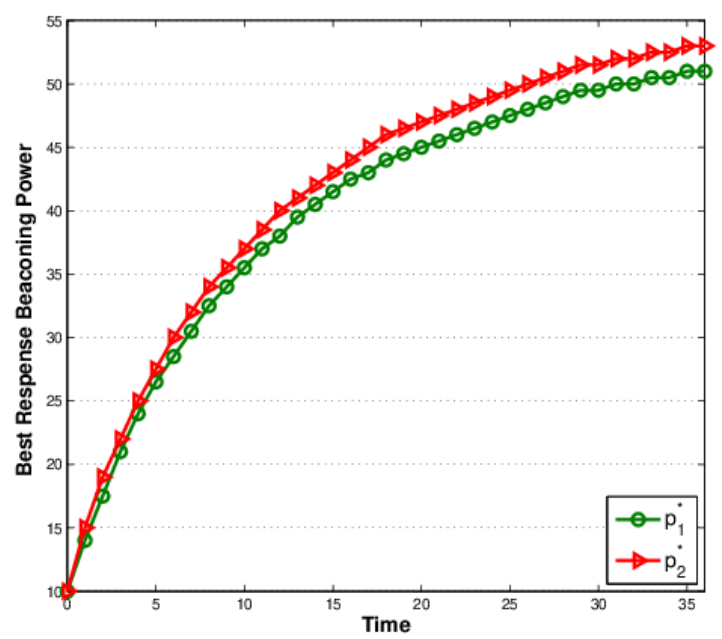

Fig. 1. Seeking the equilibrium beaconing power using the best response algorithm.

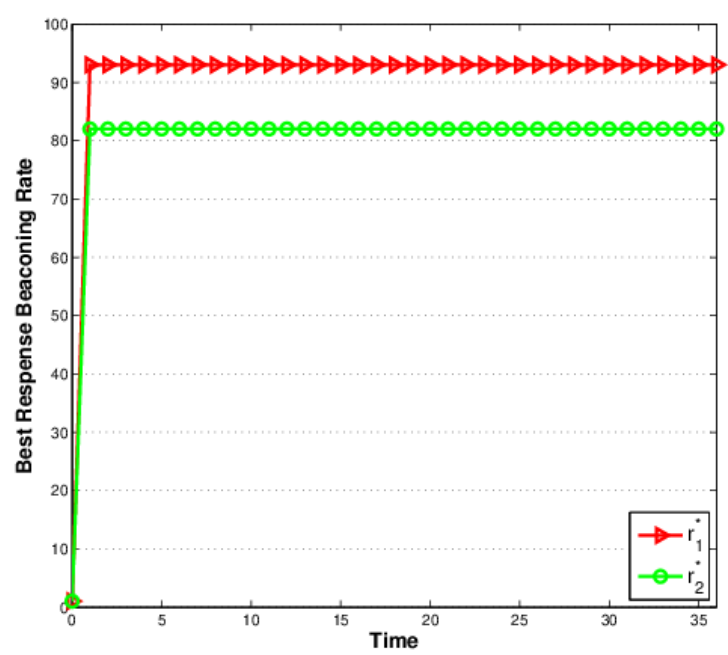

Fig. 2. Seeking the beaconing equilibrium rate using the best response algorithm.

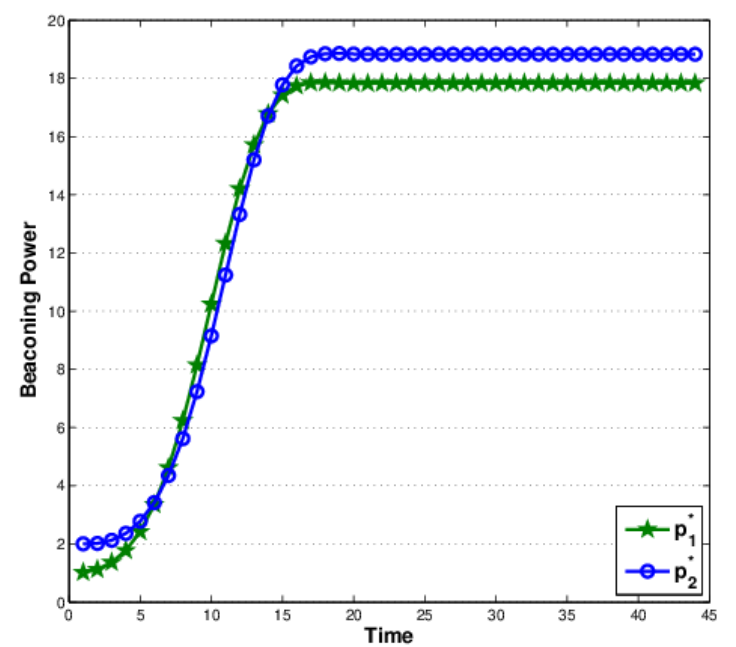

Fig. 3. Seeking the equilibrium beaconing power using the Nash seeking algorithm.

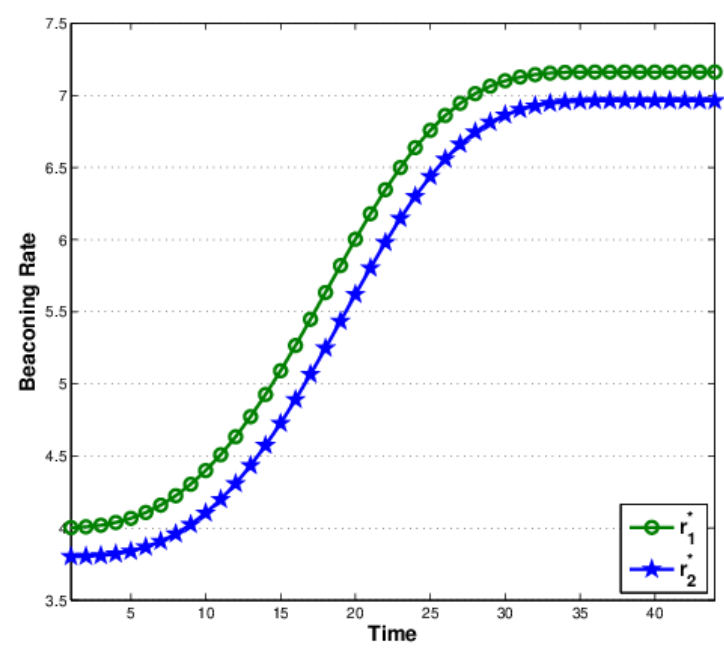

Fig. 4. Seeking the beaconing equilibrium rate using the Nash seeking algorithm.

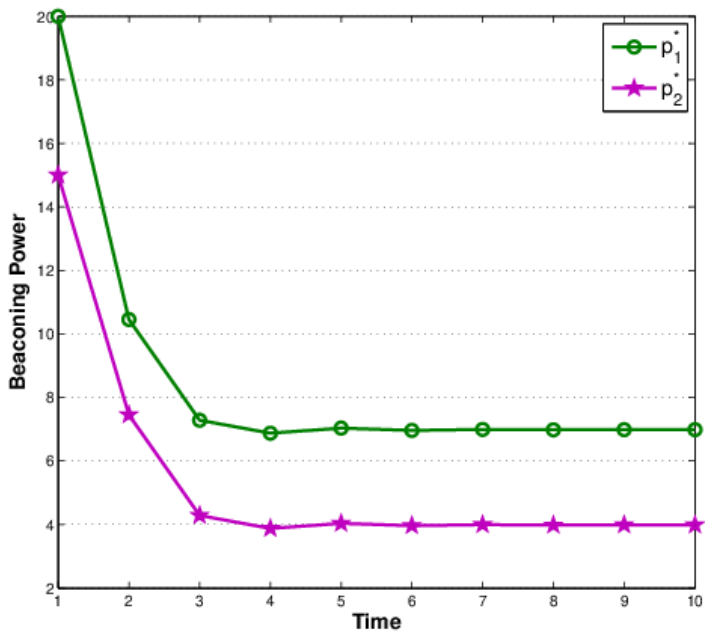

Fig. 5. Seeking the equilibrium beaconing power using a cooperative bargaining algorithm. 
Joint Beacon Power and Beacon Rate Control Based on Game Theoretic Approach in Vehicular Ad Hoc Networks

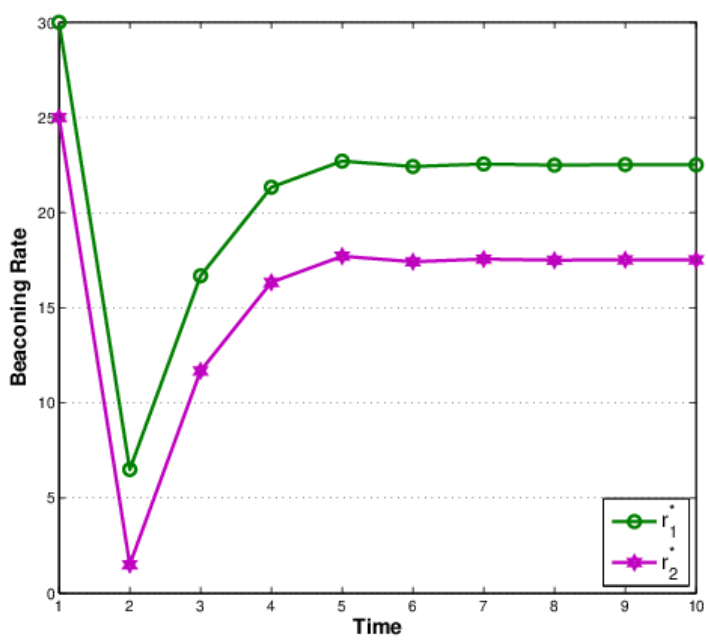

Fig. 6. Seeking the beaconing equilibrium rate using a cooperative bargaining algorithm.

The uniqueness of the joint beaconing rate and beaconing power at Nash equilibrium is demonstrated in Figures 1, 2, 3, and 4 . The best response algorithm and Nash seeking algorithm converges to the values of the beaconing rate and beaconing power at Nash equilibrium. Furthermore, based on the results presented in figure $1,2,3,4,5$, and 6 we observe that the convergence of the proposed algorithms is very fast, Nash seeking algorithm converges within approximately 43 iterations, the best response algorithm needs five to 35 iterations to converge, while the cooperative bargaining algorithm converges after 10 iterations to the Pareto-optimal equilibrium. Then, the cooperative bargaining algorithm is the algorithm that converges very fast to the equilibrium; thus, it can be easily adopted in a realistic scenario.

Note that for any vehicle $i$, it's Nash equilibrium beaconing rate $r_{i}$ and beaconing power $p_{i}$ primarily depends on the parameter $a_{i}, c_{i}, C_{p_{i}}$ and $C_{r_{i}}$. As such, we investigate how the Nash equilibrium points can be affected by these parameters.

Figures 7 and 8 show the beaconing rate and beaconing power of the vehicle when the parameter $a$ increases from 1 to 20 . The beaconing rate and beaconing power of the vehicle increase with the increase of the parameter $a$. The reason is that as the parameter $a$ increases, the utility increase. Therefore, the vehicles are more incentive to increase their beaconing rate and beaconing power. Greater parameter $a$ leads to the use of higher beaconing rate and beaconing power by vehicles because of the utility function increases.

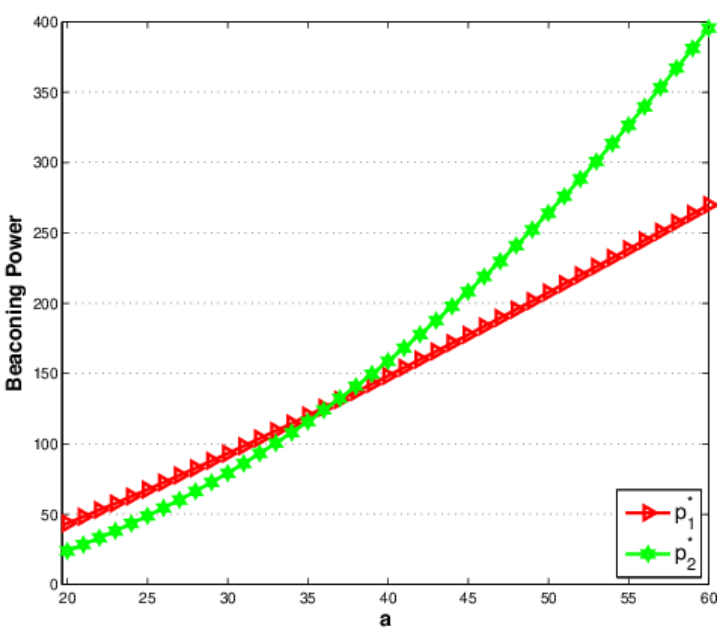

Fig. 7. Beaconing power with respect to $a$.

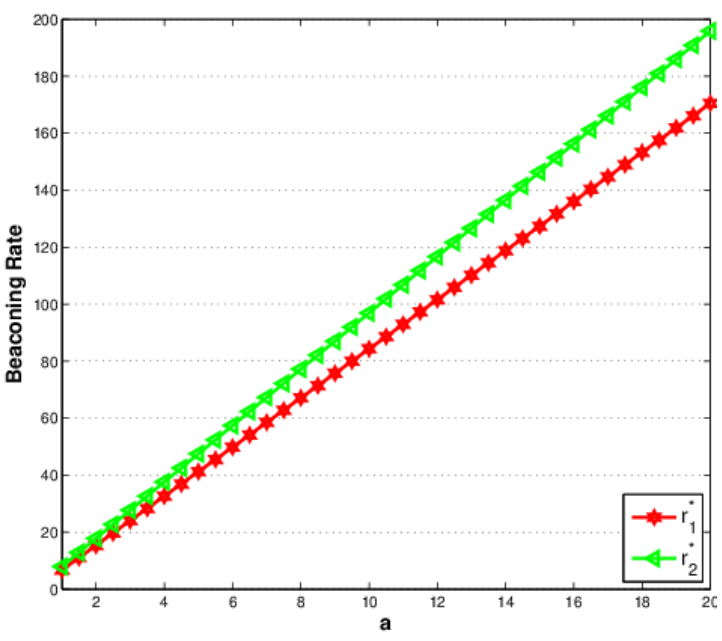

Fig. 8. Beaconing rate with respect to $a$.

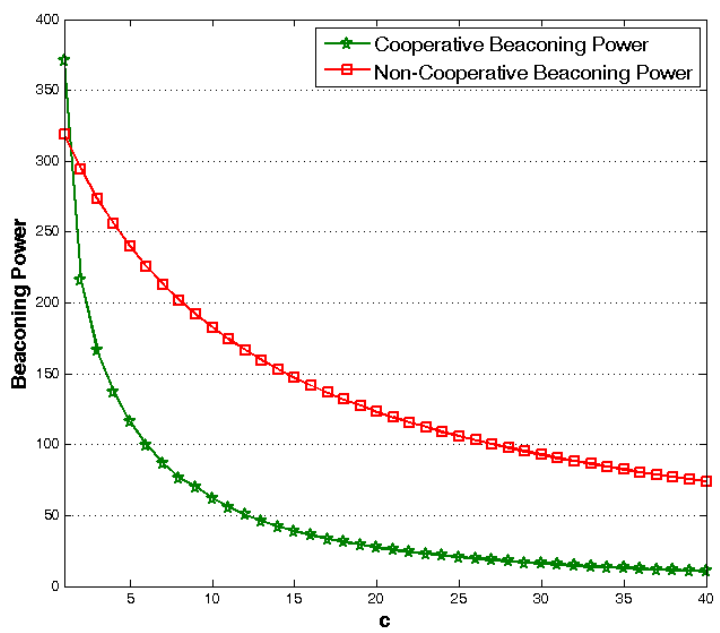

Fig. 9. Beaconing power with respect to $c$. 


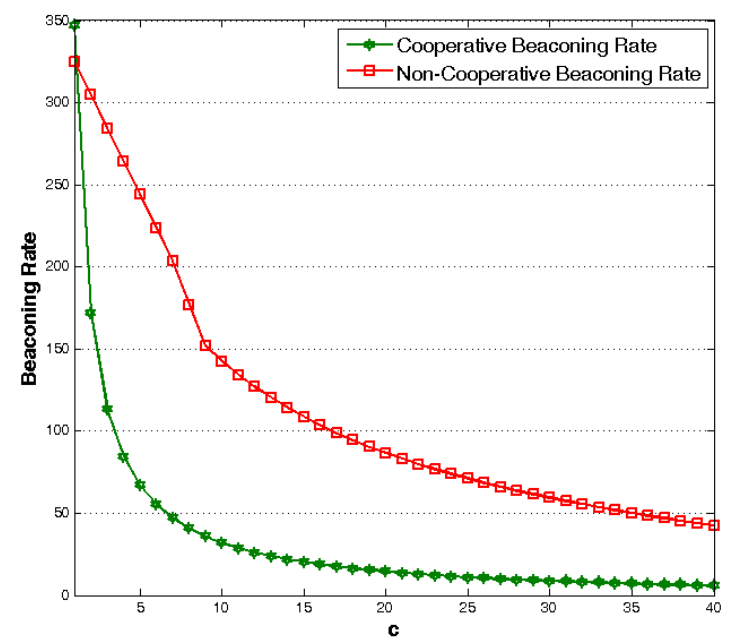

Fig. 10. Beaconing rate with respect to $c$.

We plot in Figures 9 and 10, respectively, the interplay of cost $c$ the beaconing rate and beaconing power, for both vehicles that we consider in this example. On the one hand, we note that the beaconing equilibrium rate and beaconing power for both vehicles is decreasing with respect to the cost $c$. When the cost $c$ increases, the vehicles pay more price at higher congestions, yielding a lower payoff. Therefore, the vehicles need to decrease their beaconing rate and beaconing power to decrease the congestion cost. In addition, the Nash bargaining solution beaconing strategy are lower than the non-cooperative beaconing strategy, which indicates that the Nash bargaining solution is more efficient in terms of congestion cost. Therefore, cooperation is the best choice for the vehicle.

Figures 11 and 12 show both the beaconing power and the beaconing for the non-cooperative games and the cooperative strategic beaconing obtained using the Nash bargaining solution. When energy cost $\left(C_{r}\right.$ and $\left.C_{p}\right)$ increases, the beaconing power, and beaconing rate decreases, it can be seen that the Nash bargaining solution beaconing strategy exhibits low as the energy cost level increases compared to the noncooperative beaconing strategy. A unique feature is that the strategic beaconing scheme based on the Nash bargaining solution performs better in terms of energy compared to the non-cooperative strategy for all the values of energy cost. Therefore, the Nash bargaining solution scheme guarantees a higher network lifetime compared to a non-cooperative policy.

Figure 13 shows the $P o A$ variation curve as a function of the parameter $c$. PoA decreases with respect to $c$. When $c$ is lower, the price of anarchy is socially efficient; moreover, when $c$ is lower, the vehicles cooperate for optimizing the Nash equilibrium. On the other hand, when $c$ increase the $P o A$ is lower, then the Nash equilibrium is not socially efficient, vehicles are selfish, and each one seeks to maximize its profit individually.

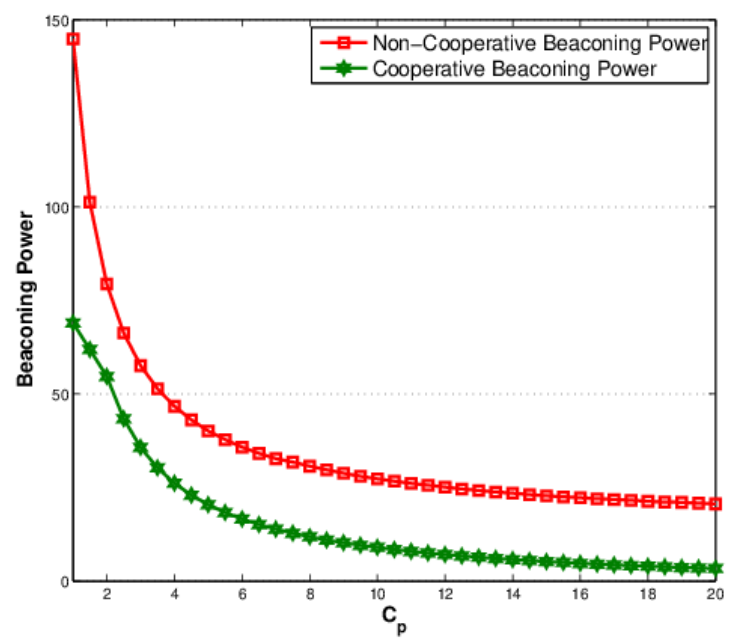

Fig. 11. Beaconing power with respect to $C_{p}$.

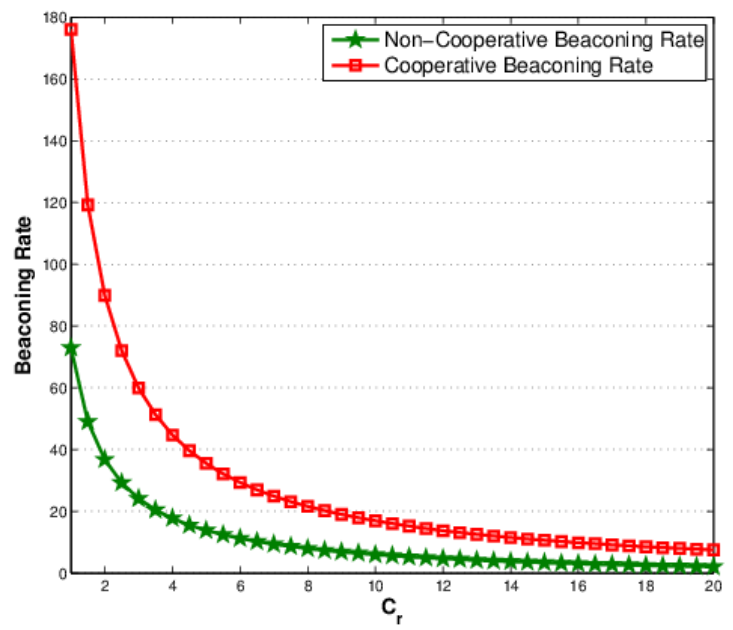

Fig. 12. Beaconing rate with respect to $C_{r}$.

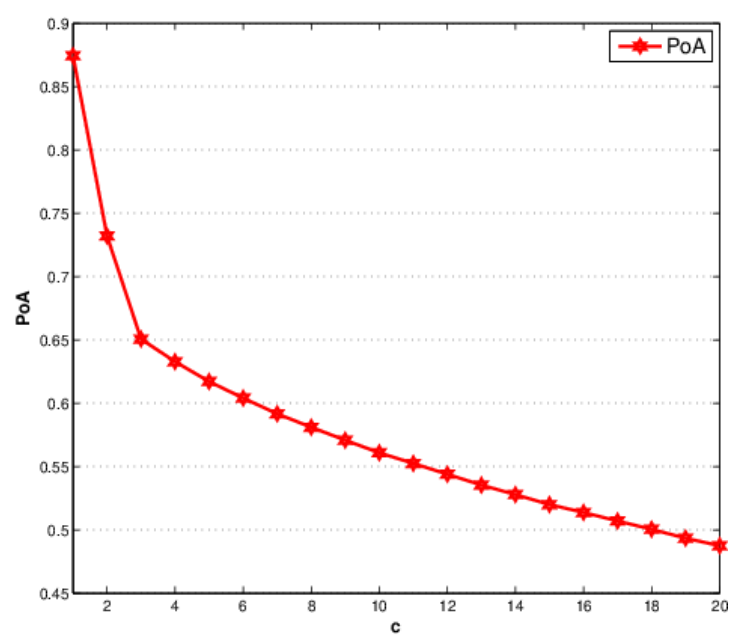

Fig. 13. Price of Anarchy as a function of parameter $c$. 


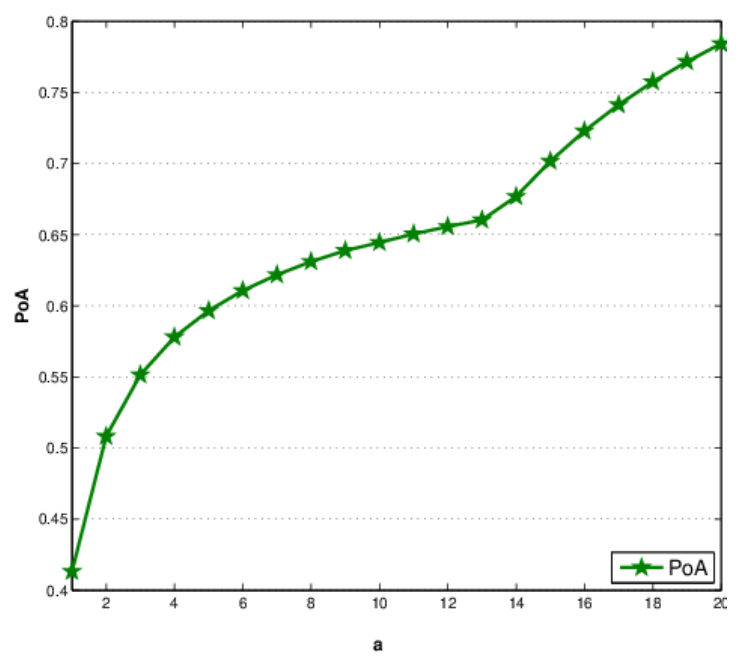

Fig. 14. Price of Anarchy as a function of parameter $a$.

Figure 14 shows $P o A$ variation curve as a function of parameter $a$. In that figure, $P o A$ increases with respect to the parameter $a$. When the parameter $a$ is lower, the price of anarchy is lower. Then, the Nash equilibrium is not socially efficient, the vehicles are selfish, and each one seeks to maximize its profit individually. However, when the parameter $a$ increases, the equilibrium becomes more and more socially efficient, this increase finds the simple intuition that when parameter $a$ increase vehicles cooperate with each other for optimizing Nash equilibrium.

\section{CONCLUSION}

In this paper, the problem of joint beaconing rate and beaconing power control in VANETs is addressed via S-modular theory. The competition between the vehicle in VNETs is formulated as a non-cooperative game and a cooperative game, where each vehicle chooses the joint beaconing rate and beaconing power. We have performed the equilibrium analysis and proposed a three distributed algorithm for computing the equilibrium point. Simulation results illustrate the impacts of the system parameters on the joint beaconing rate and beacon power and show the number of iteration required by each algorithm for the convergence to the equilibrium. The analysis and simulation results provide a better understanding of the complex interactions among vehicles under a competitive and cooperative condition, which is a benefit for the optimization of vehicle strategies.

\section{REFERENCES}

[1] J. E. Paquet, "Guidance for Intelligent Transport Systems (ITS) in Urban Areas", No March, 2010.

[2] D.-M. Chiu et R. Jain, "Analysis of the increase and decrease algorithms for congestion avoidance in computer networks", Comput. Netw. ISDN Syst., vol. 17, no 1, p. 1-14, 1989.

[3] M. Outanoute, H. Garmani, M. Baslam, R. El Ayachi, et B. Bouikhalene, "A Non-Cooperative Game Analysis of Competition between Content Providers in the Internet Market":, Int. J. Bus. Data Commun. Netw., vol. 15, no 1, p. 88-104, janv. 2019, DoI: $10.4018 / \mathrm{IJBDCN} .2019010106$
[4] D. A. Omar, H. Garmani, M. El Amrani, M. Baslam, et M. Fakir, "Analysis of Bargaining Game Policy in the Internet Content Distribution Chain":, Int. J. Mob. Comput. Multimed. Commun., vol. 10, no 3, p. 47-73, juill. 2019, Dor: 10.4018/IJMCMC.2019070103.

[5] D. A. Omar, H. Garmani, M. El Amrani, M. Baslam, et M. Fakir, "Analyzing the Customers' Dynamic Confusion in Telecommunication Networks Share Game:", Int. J. Bus. Data Commun. Netw., vol. 15, no 2, p. 15-34, juill. 2019, Dor: 10.4018/IJBDCN.2019070102.

[6] H. Garmani, M. Outanoute, M. Baslam, et M. Jourhmane, "New Competition-Based Approach for Caching Popular Content in ICN", in Networked Systems, vol. 11028, A. Podelski et F. Taïani, Éd. Cham: Springer International Publishing, 2019, p. 286-300.

[7] H. Garmani, M. Baslam, et M. Jourhmane, "Analysis of Competition between CPs Fronting Advertisers", Int. J. Adv. Sci. Technol., vol. 117, p. 53-66, août 2018, Dor: 10.14257/ijast.2018.117.05.

[8] D. Ait Omar, M. Outanoute, M. Baslam, M. Fakir, et B. Bouikhalene, "On understanding price-QoS war for competitive market and confused consumers", Computing, vol. 101, no 9, p. 1327-1348, sept. 2019, Dor: $10.1007 / \mathrm{s} 00607-018-0642-5$.

[9] L. Le, R. Baldessari, P. Salvador, A. Festag, et W. Zhang, "Performance evaluation of beacon congestion control algorithms for VANETs", in 2011 IEEE Global Telecommunications Conference-GLOBECOM 2011, 2011, p. 1-6.

[10] F. Li et C. Huang, "A Mobility Prediction Based Beacon Rate Adaptation Scheme in VANETs", in 2018 IEEE Symposium on Computers and Communications (ISCC), Natal, juin 2018, p. 0067100677, DoI: 10.1109/ISCC.2018.8538734.

[11] H. P. Luong, M. Panda, H. Le Vu, et Q. B. Vo, "Analysis of multi-hop probabilistic forwarding for vehicular safety applications on highways", IEEE Trans. Mob. Comput., vol. 16, no 4, p. 918-933, 2017.

[12] K. N. Qureshi, A. H. Abdullah, O. Kaiwartya, S. Iqbal, R. A. Butt, et F. Bashir, "A Dynamic Congestion Control Scheme for safety applications in vehicular ad hoc networks", Comput. Electr. Eng., vol. 72, p. 774- 788, nov. 2018, Dor: 10.1016/j.compeleceng.2017.12.015.

[13] M. Ishaq, M. H. Malik, et M. E. Aydin, "Managing Congestion in Vehicular Networks Using Tabu Search", in Engineering Applications of Neural Networks, vol. 893, E. Pimenidis et C. Jayne, Éd. Cham: Springer International Publishing, 2018, p. 118-129.

[14] F. Goudarzi et H. Asgari, "Non-Cooperative Beacon Power Control for VANETs", IEEE Trans. Intell. Transp. Syst., p. 1-6, 2018, Dor: 10.1109 /TITS.2018.2813258.

[15] F. Goudarzi et H. Asgari, "Non-Cooperative Beacon Rate and Awareness Control for VANETs", IEEE Access, vol. 5, p. 1685816870, 2017, Dor: 10.1109/ACCESS.2017.2742864.

[16] Q. Chen, D. Jiang, T. Tielert, et L. Delgrossi, "Mathematical Modeling of Channel Load in Vehicle Safety Communications", in 2011 IEEE Vehicular Technology Conference (VTC Fall), San Francisco, CA, USA, sept. 2011, p. 1-5, Dor: 10.1109/VETECF.2011.6093282.

[17] L. Guijarro, V. Pla, J. R. Vidal, et J. Martinez-Bauset, "Analysis of price competition under peering and transit agreements in Internet Service provision to peer-to-peer users", in 2011 IEEE Consumer Communications and Networking Conference (CCNC), janv. 2011, p. 1145-1149, Dor: $10.1109 / \mathrm{CCNC} .2011 .5766356$.

[18] J. F. Nash Jr, "The bargaining problem", Econom. J. Econom. Soc., p. 155-162, 1950.

[19] D. Fudenberg et Tirole, J., Game Theory. The MIT Press, Cambridge, Massachusetts, 1993.

[20] C. Shi, S. Salous, F. Wang, et J. Zhou, "Power allocation for target detection in radar networks based on low probability of intercept: A cooperative game theoretical strategy", Radio Sci., vol. 52, no 8, p. $1030-1045,2017$. 
Joint Beacon Power and Beacon Rate Control Based on Game Theoretic Approach in Vehicular Ad Hoc Networks

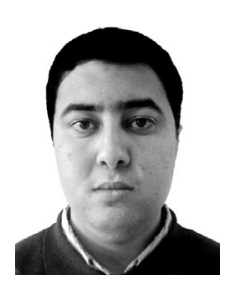

Hamid Garmani received the Ph.D. degrees from University Sultan Moulay Slimane, Morocco, in 2020. His research interests include network economics, network security, applications of game theory in wireless networks, and radio resource management.

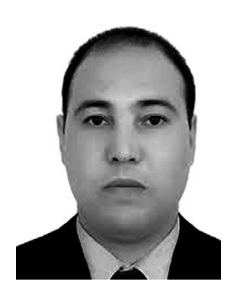

Driss Ait Omar received the Ph.D. degrees from University Sultan Moulay Slimane, Morocco, in 2019. His research interests include network economics, applications of game theory in wireless networks, network security.

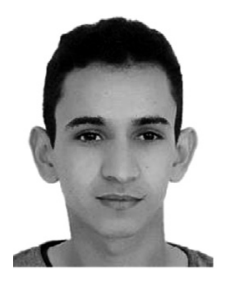

Mohamed El Amrani is a PhD student at the Faculty of Sciences and Technology, University Sultan Moulay Slimane, Morocco. His research interests include network economics, network security, applications of game theory in wireless networks, and radio resource management.

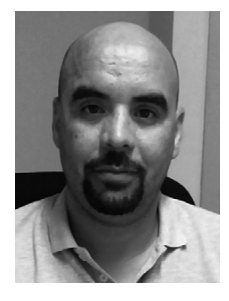

Mohamed Baslam is a Professor of computer science in the Faculty of Sciences and Technology, Sultan Moulay Slimane University, Morocco. His current research interests include performance evaluation and optimization of networks based on game-theoretic and queuing models, applications in communication/ transportation and social networks, such as wireless flexible networks, bio-inspired and self-organizing networks, and economic models of the Internet and yield management.

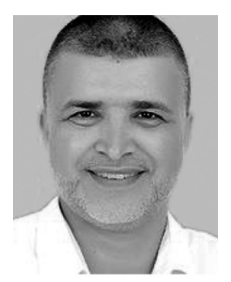

Mostafa Jourhmane is a professor of mathematics and computer science in the Faculty of Sciences and Technology, Sultan Moulay Slimane University, Morocco. His research interests include Numerical analysis, classification, image processing, neural networks, big data, Network Engineering Games, social networks and their control and the analysis through game theoretical models of network and neutrality issues. 